Article

\title{
Anthocyanin Accumulation in the Leaves of the Purple Sweet Potato (Ipomoea batatas L.) Cultivars
}

\author{
GuoLiang Li®, Zhaomiao Lin, Hong Zhang, Zhonghua Liu, Yongqing Xu, Guochun Xu, \\ Huawei Li, Rongchang Ji, Wenbin Luo, Yongxiang Qiu, Sixin Qiu * and Hao Tang
}

Institute of Crop Sciences, Fujian Academy of Agricultural Sciences, Scientific Observing and Experimental Station of Tuber and Root Crops in South China, Ministry of Agriculture. Fuzhou, Fujian 350013, China; uslg1@126.com (G.L.); linzhaomiao@foxmail.com (Z.L.); teeteeking@163.com (H.Z.); lh18620@163.com (Z.L.); qingqing0722@126.com (Y.X.); xgc_faas@163.com (G.X.); fjpotato@126.com (H.L.); jrc1976@163.com (R.J.); lwb9630@163.com (W.L.); qyxlm@sohu.com (Y.Q.); tanghao9403@163.com (H.T.)

* Correspondence: qiusixin@faas.cn; Tel.: +86-0591-87572407

Received: 20 September 2019; Accepted: 15 October 2019; Published: 17 October 2019

\begin{abstract}
Sweet potato anthocyanins are water-soluble pigments with many physiological functions. Previous research on anthocyanin accumulation in sweet potato has focused on the roots, but the accumulation progress in the leaves is still unclear. Two purple sweet potato cultivars (Fushu No. 23 and Fushu No. 317) with large quantities of anthocyanin in the leaves were investigated. Anthocyanin composition and content were assessed with ultra-performance liquid chromatography diode-array detection (UPLC-DAD) and ultra-performance liquid chromatography/quadrupole time-of-flight mass spectrometry (UPLC-QTOF-MS), and the expressions of genes were detected by qRT-PCR. The two cultivars contained nine cyanidin anthocyanins and nine peonidin anthocyanins with an acylation modification. The acylation modification of anthocyanins in sweet potato leaves primarily included caffeoyl, $p$-coumaryl, feruloyl, and $p$-hydroxy benzoyl. We identified three anthocyanin compounds in sweet potato leaves for the first time: cyanidin 3-p-coumarylsophoroside-5-glucoside, peonidin 3-p-coumarylsophoroside-5-glucoside, and cyanidin 3-caffeoyl-p-coumarylsophoroside-5-glucoside. The anthocyanidin biosynthesis downstream structural genes DFR4, F3H1, anthocyanin synthase (ANS), and UDP-glucose flavonoid 3-O-glucosyltransferase (UFGT3), as well as the transcription factor MYB1, were found to be vital regulatory genes during the accumulation of anthocyanins in sweet potato leaves. The composition of anthocyanins (nine cyanidin-based anthocyanins and nine peonidin-based anthocyanins) in all sweet potato leaves were the same, but the quantity of anthocyanins in leaves of sweet potato varied by cultivar and differed from anthocyanin levels in the roots of sweet potatoes. The anthocyanidin biosynthesis structural genes and transcription factor together regulated and controlled the anthocyandin biosynthesis in sweet potato leaves.
\end{abstract}

Keywords: sweet potato; anthocyanin compositions; biosynthesis structural genes; transcription factor

\section{Introduction}

The sweet potato (Ipomoea batatas L.) is an important tropical crop, providing starch, beta-carotene, and anthocyanins for human nutrition and industrial use [1]. Leafy sweet potato is a new type in China that is consumed for its stems and leaves, not the tuberous root. The stems and leaves are nutritious and contain high levels of protein, dietary fiber, calcium, magnesium, iron, zinc, flavonoids, and anthocyanins [2]. In 2003, the first leafy sweet potato cultivar was cultivated in China, called Fushu No. 7-6 [3], but in 2016 there was only one purple leafy sweet potato cultivar being grown in China, Fushu No. 23. Fushu No. 23 has been found to have high levels of anthocyanin in both the stems and leaves (Figure 1). 


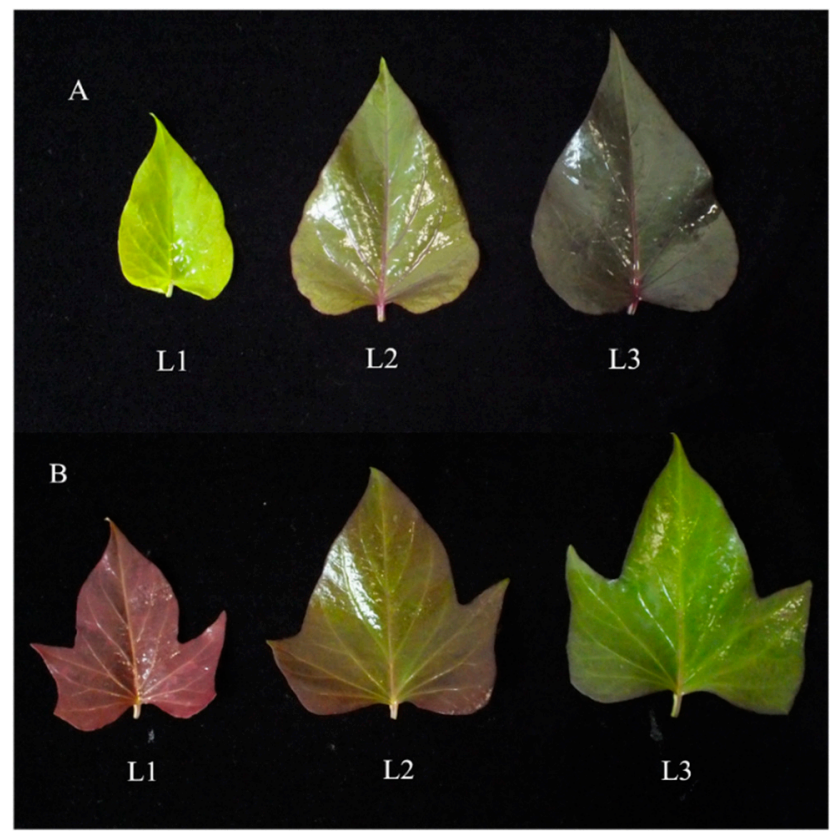

Figure 1. Photographs of sweet potato leaves with differential levels of accumulated anthocyanins. (A) Fushu No. 23, (B) Fushu No. 317, "L1" indicates the primary leaves (three days old), "L2" indicates the second leaves (five days old), and "L3" indicates the third leaves (seven days old).

Anthocyanins are a group of water-soluble natural pigments that are considered flavonoids with a basic structure of C6-C3-C6. They are responsible for fruit and flower coloration [4]. Previous research on anthocyanins in sweet potato has focused on the roots and found that the basic anthocyanin monomers are cyanidin, peonidin, and pelargonidin. These anthocyanin monomers are often linked to acylated glucoside or sophoroside, and acylation modification comes from caffeic acid, $p$-coumaric acid, ferulic acid, and $p$-hydroxybenzoic acid [4-8]. Terahara et al. (1999) identified eight acylated anthocyanins in the roots of the purple Japanese sweet potato cultivar Yamagawamurasaki, and six monomers were identified as diacylated anthocyanins [9]. Tian et al. (2005) identified 26 anthocyanins from the 'Ayamurasaki' line that had been root cultured, and it was first discovered that the purple-fleshed sweet potato contained pelargonidin 3-sophoroside-5-glucoside and pelargonidin 3-feruloylsophoroside-5-glucoside [4]. Lee et al. (2013) separated six pelargonidin-based anthocyanins from Korean purple-fleshed sweet potato cultivar Borami [6], and found that compared with the purple-fleshed root, anthocyanins in sweet potato leaves were relatively less studied. Islam et al. (2002) detected 15 anthocyanin compounds from sweet potato leaves, and eight cyanidin derivatives were identified and measured [10]. Su et al. (2019) identified 14 anthocyanins with a new anthocyanin peonidin 3-caffeoyl-p-coumaryl sophoroside-5-glucoside in the leaves of three sweet potato varieties [11]. Vishnu et al. (2019) found that peonidin derivatives were the major anthocyanins in tubers and the leaves, but that the contents of the cyanidin derivatives were greater in leaves than in tubers [12]. Studies have shown that anthocyanins from sweet potato have good properties regarding scavenging 1,1-diphenyl-2-picrylhydrazyl (DPPH) radicals [13], and protect the liver [14,15], have anti-tumor properties [16], lower blood sugar levels [17], and have other positive physiological functions on the human body $[18,19]$.

In this study, two purple sweet potato cultivars (Fushu No. 23 and Fushu No. 317) with different patterns of anthocyanin accumulation were studied (Figure 1). The content and chemical structures of anthocyanin monomers in the leaves were analyzed and the expression of anthocyanin biosynthetic structural genes and transcription factors were studied. 


\section{Materials and Methods}

\subsection{Chemicals}

All of the reagents or solvents were of analytical, HPLC, or HPLC-MS grade. Absolute ethanol, acetone, hydrochloric acid $(\mathrm{HCl})$, and sodium hydroxide $(\mathrm{NaOH})$ were obtained from Sinopharm Chemical Reagent Co., Ltd. (Shanghai, China). Methanol and ethanol in LC-MS grade was purchased from Honeywell (Muskegon, MI, USA). Acetonitrile in LC-MS grade was obtained from Merck KgaA (Darmstadt, Germany). Formic acid in LC-MS grade was the product of Fisher Scientific (Waltham, MA, USA). The standard cyanidin 3-O-glucoside was purchased from Sigma-Aldrich (St. Louis, MO, USA). The TransStart Top Green qPCR SuperMix was purchased from Transgen Biotech (Beijing, China).

\subsection{Plant Materials}

The purple-leaf sweet potato cultivars Fushu No. 23 and Fushu No. 317 were sampled under natural outdoor light and temperature conditions. Two-thirds of samples were stored at $-80{ }^{\circ} \mathrm{C}$ in a refrigerator (Haier Group Co., Qingdao, China) for later analyses of metabolites. To determine the content of total anthocyanins, the remaining $1 / 3$ of leaves were oven-dried, ground into powder with a diameter less than $0.3 \mathrm{~mm}$, and stored in a vacuum pack (VIP320, Beijing Torch SMT Inc. Co., Beijing, China) at $4{ }^{\circ} \mathrm{C}$.

In order to understand the difference in anthocyanin levels between roots and leaves, some purple-fleshed sweet potatoes were examined, the cultivars Fushu No. 9, Fushu No. 24, Fushu No. 317, Ornamental Purple (OP), Fushu No. 23, and Fushu No. 25 were obtained from the Institute of Crop Sciences, Fujian Academy of Agricultural Sciences, Fuzhou, China, (26 $08^{\prime}$ N, $119^{\circ} 28^{\prime}$ E) in August 2018. Yanshu No. 5, Longzishu No. 6, and Longzishu No. 8, were generously provided by the Longyan Institute of Agricultural Sciences, Longyan, China, in August 2018.

\subsection{Sample Preparation}

Two-thirds of lyophilized samples (50 mg) were grounded by TissueLyser JX-24 (Jingxin, Shanghai, China) with beads at $40 \mathrm{~Hz}$ for $4 \mathrm{~min}$, and extracted with $0.5 \mathrm{~mL}$ of $70 \%$ methanol containing $0.1 \%$ formic acid. Samples were then processed for $10 \mathrm{~min} 100 \mathrm{HZ}$ ultra-sonication in ice water. The mixtures were vortexed for $30 \mathrm{~s}$ and left to stand for $2 \mathrm{~h}$ at $-40{ }^{\circ} \mathrm{C}$. After, samples were centrifuged at $4{ }^{\circ} \mathrm{C}$ at 14,000 rpm for $15 \mathrm{~min}$, and $350 \mu \mathrm{L}$ of supernatant were dried under gentle nitrogen stream and re-dissolved in $90 \mu \mathrm{L}$ of $70 \%$ methanol containing $0.1 \%$ formic acid combined with $10 \mu \mathrm{L}$ of $25 \mu \mathrm{g} / \mathrm{mL}$ lidocaine (internal standard) prior to ultra performance liquid chromatography/quadrupole time-of-flight mass spectrometry (UPLC-QTOF-MS) analysis. Quality control (QC) sample was obtained by isometrically mixing the prepared samples. The injection volume was $1 \mu \mathrm{L}\left(\mathrm{ESI}^{+}\right)$.

\subsection{Ultra Performance Liquid Chromatography Diode-Array Detection (UPLC-DAD) and UPLC-QTOF-MS Analysis}

Chromatographic separation was performed on an ACQUITY UPLC I-Class system (Waters Corporation, Milford, MA, USA) with an ACQUITY UPLC BEH C18 column $(100 \times 2.1 \mathrm{~mm}, 1.7 \mu \mathrm{m}$, Waters Corporation, Milford, MA, USA) maintained at $45{ }^{\circ} \mathrm{C}$. The injection volume was $3 \mu \mathrm{L}$. The mobile phases consisted of water (phase A) and acetonitrile (phase B), both with $0.5 \%$ formic acid $(v / v)$. A linear gradient elution was performed using the following program: $0-2 \mathrm{~min}, 1 \% \mathrm{~B} ; 3 \mathrm{~min}, 5 \% \mathrm{~B} ; 9$ $\min , 20 \%$ B; $12 \mathrm{~min}, 50 \% \mathrm{~B} ; 15 \mathrm{~min}, 100 \% \mathrm{~B} ; 17 \mathrm{~min}, 100 \% \mathrm{~B} ; 17.1 \mathrm{~min}, 1 \% \mathrm{~B}$, and held for $20 \mathrm{~min}$.

The eluents were analyzed on a Vion IMS QTOF Mass spectrometer (Waters Corporation, Milford, MA, USA) set to $\mathrm{ESI}^{+}$mode. The capillary voltage was set to $2 \mathrm{kV}$. The sampling cone voltage and cone gas flow were $40 \mathrm{~V}$ and $50 \mathrm{~L} / \mathrm{h}$, respectively. The desolvation gas was maintained at a flow rate of $900 \mathrm{~L} / \mathrm{h}$ and a temperature of $450{ }^{\circ} \mathrm{C}$. The ion source temperature was $115^{\circ} \mathrm{C}$. The TOF-MS scan was operated at a high-resolution with $0.2 \mathrm{~s}$ survey scan time and a range of $50-1000 \mathrm{~m} / \mathrm{z}$ in the continuum mode for both function 1 and 2. To improve the identification of unknown metabolites, $\mathrm{MS}^{\mathrm{E}}$ function 
was also performed to obtain fragment ion information with a ramp collision energy from 20 to $45 \mathrm{eV}$. The mass accuracy calibration was performed with the $250 \mathrm{ng} / \mathrm{mL}$ lock mass leucine-enkephalin at 5 $\mu \mathrm{L} / \mathrm{min}$, with data acquisition frequency set at $30 \mathrm{~s}$. The software for controlling the instrument and collecting data was UNIFI 1.8.1 (Waters Corporation, Milford, MA, USA). Peak picking, alignment, and deconvolution were conducted using Progenesis QI (Nonlinear Dynamics, Newcastle, UK) with default parameters. A suitable quality control sample (QC from pooled samples) from the run was selected as a reference for peak alignment. The structural identification of anthocyanins was performed using UNIFI 1.8.1(Waters Cooperation, Milford, MA, USA). The anthocyanin content was expressed as mg of cyanidin 3-O-glucoside equivalent.

\subsection{Quantitative Real-time PCR ANALYSIS of Anthocyanin Biosynthetic Genes in Sweet Potato Leaves}

Total RNA was isolated from sweet potato leaves in September using TransZol Plant (Transgen Biotech Inc., Beijing, China) following the manufacturer's instructions. First-strand cDNA were reverse transcribed using a Reverse Transcriptase M-MLV Kit (Promega, Madison, WI, USA). The qRT-PCR assay was performed on an ABI QuantStudio5 Real-time system (ABI, Foster City, CA, USA), and in $10 \mu \mathrm{L}$ of reaction containing 2× TransStart Top Green qPCR SuperMix (Transgen Biotech Inc., Beijing, China), $10 \mu \mathrm{M}$ of solution from each primer (Supplementary Table S1), and $100 \mathrm{ng}$ of cDNA. Thermocycling conditions were: initial denaturation at $95^{\circ} \mathrm{C}$ for $2 \mathrm{~min}$, followed by 40 cycles for $15 \mathrm{~s}$ at $95^{\circ} \mathrm{C}$, and $1 \mathrm{~min}$ at $60^{\circ} \mathrm{C}$. The relative expression of anthocyanin biosynthetic genes was assessed by the comparative threshold cycle (Ct) method [20]. The sweet potato actin gene (NCBI accession: EU250003) served as an internal control for signal normalization. Expression levels were evaluated as technical duplicates of biological triplicates from separate plant samples.

\section{Results and Discussion}

\subsection{Total Monomeric Anthocyanin Content in the Leaves of the Cultivars Fushu No. 23 and Fushu No. 317}

To further understand the differences between the two purple sweet potato cultivars in the accumulation of anthocyanins, the composition and content of anthocyanins were detected by UPLC-DAD and UPLC-QTOF-MS (Figure 2, Tables 1 and 2). Eighteen anthocyanin monomers were found, which contained a typical anthocyanin spectrum of a maximum absorbance at $520 \mathrm{~nm}$.

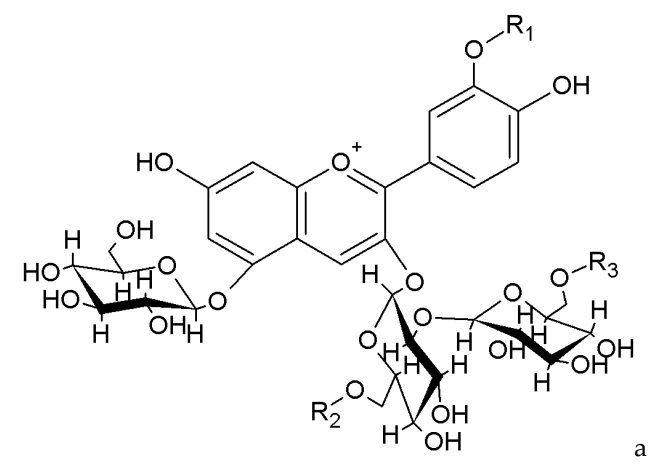<smiles>O=C(O)/C=C/c1ccc(O)c(O)c1</smiles>

b<smiles>COc1cc(/C=C/C(=O)O)ccc1O</smiles><smiles>O=C(O)c1ccc(O)cc1</smiles><smiles>O=C(O)/C=C/c1ccc(O)cc1</smiles>

$\mathrm{d}$

Figure 2. Skeleton structures of anthocyanins in sweet potato leaves. (a) Skeleton structures of anthocyanins; (b) caffeic acid ( $m / z$ 180); (c) ferulic acid ( $\mathrm{m} / \mathrm{z} 194) ;(\mathbf{d}) \mathrm{p}$-hydro benzoic acid $(\mathrm{m} / \mathrm{z} 138)$; and (e) $p$-coumaroyl acid $(m / z$ 164). 
Table 1. Identification of anthocyanins (1-14) from cultivar Fushu No. 23 and cultivar Fushu No. 317 leaves using ultra performance liquid chromatography/quadrupole time-of-flight mass spectrometry (UPLC-QTOF-MS/MS).

\begin{tabular}{|c|c|c|c|c|c|c|}
\hline \multirow{2}{*}{$\begin{array}{c}\text { Peak } \\
1\end{array}$} & \multirow{2}{*}{$\frac{\mathbf{R t}^{\mathbf{a}}(\mathbf{m i n})}{4.45}$} & \multicolumn{3}{|c|}{ Fragment Ions $(m / z)$} & \multirow{2}{*}{$\begin{array}{c}{[\mathbf{M}]^{+}(\mathrm{m} / \mathrm{z})} \\
773.2117\end{array}$} & \multirow{2}{*}{$\begin{array}{c}\text { Identity }^{\mathbf{b}} \\
\text { Cy 3-soph-5glc }\end{array}$} \\
\hline & & 287 & 449 & 611 & & \\
\hline 2 & 4.99 & 301 & 463 & 625 & 787.2266 & Peo 3-soph-5glc \\
\hline 3 & 5.92 & 287 & 449 & 731 & 893.2325 & Cy 3-p-hydroxybenzoylsoph-5glc \\
\hline 4 & 6.10 & 287 & 449 & 773 & 935.2421 & Cy 3-caffeylsophsoph-5glc \\
\hline 5 & 6.63 & 301 & 625 & 745 & 907.2479 & Peo 3-p-hydroxybenzoylsoph-5glc \\
\hline 6 & 6.77 & 301 & 463 & 787 & 949.2578 & Peo 3-caffeylsophsoph-5glc \\
\hline 7 & 6.86 & 287 & 449 & 757 & 919.2477 & Cy 3-p-coumarylsoph-5glc \\
\hline 8 & 6.98 & 287 & 449 & 787 & 949.2577 & Cy 3-feruloylsoph-5glc \\
\hline 9 & 7.50 & 301 & 463 & 771 & 933.2634 & Peo 3-p-coumarylsoph-5glc \\
\hline 10 & 7.64 & 301 & 463 & 801 & 963.2734 & Peo 3-feruloylsoph-5glc \\
\hline $11 \mathrm{a}$ & 7.83 & 287 & 449 & 935 & 1097.2741 & Cy 3-dicaffeylsoph-5glc \\
\hline $11 b$ & 7.89 & 287 & 449 & 893 & 1055.2636 & Cy 3-caffeoyl-p-hydroxybenzoylsoph-5glc \\
\hline $12 \mathrm{a}$ & 8.33 & 287 & 449 & 949 & 1081.2794 & Cy 3-caffeoyl-p-coumarylsoph-5glc \\
\hline $12 b$ & 8.35 & 287 & 433 & 919 & 1111.2898 & Cy 3-caffeoyl-feruloylsoph-5glc \\
\hline $13 a$ & 8.52 & 301 & 463 & 907 & 1111.2898 & Peo 3-dicaffeoylsoph-5glc \\
\hline $13 b$ & 8.62 & 301 & 463 & 949 & 1069.2791 & Peo 3-caffeoyl-p-hydroxybenzoylsoph-5glc \\
\hline $14 \mathrm{a}$ & 9.05 & 301 & 463 & 933 & 1095.2942 & Peo 3-caffeoyl-p-coumarylsoph-5glc \\
\hline $14 \mathrm{~b}$ & 9.05 & 301 & 463 & 963 & 1125.3038 & Peo 3-caffeoyl-feruloylsoph-5glc \\
\hline
\end{tabular}

The $m / z$ ratio of the 18 anthocyanin monomers with daughter fragments was captured within the scanning interval range. Nine cyanidin was detected at $m / z 287$ and nine peonidin was detected at $\mathrm{m} / \mathrm{z}$ 301. Cyanidin 3-sophoroside-5-glucoside (Table 1, Figure S1.1, $\mathrm{m} / \mathrm{z} 773$ ) produced three fragments located at $m / z 611,449$, and 287. Transition $773>611$ and $773>449$ represented the loss of glucose $(\mathrm{m} / \mathrm{z} 162)$ and sophorose $(\mathrm{m} / \mathrm{z} 324)$, respectively, while transition $773>287$ produced cyanidin $(\mathrm{m} / \mathrm{z} 287)$ aglycone due to the loss of both glucose and sophoroside. Another example for mono- and di-acylated anthocyanin was cyanidin 3-(6"-p-hydroxybenzoylsoph)-5-glucoside (Table 1, Figure S1.3, $m / z$ 893). The transitions from 893 to 731 indicated a loss of glucose $[\mathrm{M}-162]^{+}$, and from 893 to $449[\mathrm{M}-162 \times 2-120]^{+}$ indicated a loss of sophoroside and $p$-hydroxybenzoic acid 120 [ $p$-hydroxybenzoic acid-H2O $]^{+}$. The remaining anthocyanins were identified in a similar fashion using the LC-MS library and identification results of Tian and Lee et al. [4,6] The $m / z$ ratios of each intact anthocyanin and its daughter fragments are summarized in Table 1. We identified four anthocyanin compounds that were found in the leaves of sweet potato for the first time: cyanidin 3-p-coumarylsophoroside-5-glucoside, peonidin 3-p-coumarylsophoroside-5-glucoside, cyanidin 3-caffeoyl- $p$-coumarylsophoroside-5-glucoside, and peonidin 3-caffeoyl- $p$-coumarylsophoroside-5-glucoside.

The composition of anthocyanins in the sweet potato leaves had certain regularity. The first anthocyanin monomers was cyanidin, another anthocyanin monomer peonidin was formed by methylation at the cyanidin R1 position, and other monoacylanthocyanins or diacylanthocyanins were formed by acylation modification at cyanidin or peonidin at the R2 and R3 positions [4-7]. The structure of the acylated anthocyanins was more stable and had stronger physiological activities than nonacylated anthocyanins. The acylation modification of anthocyanin in sweet potato leaves primarily included: caffeoyl, $p$-coumaroyl, feruloyl, and p-hydroxy benzoyl (Figure 2). 
Table 2. Anthocyanin monomer (1-14) content in cultivar Fushu No. 23 and cultivar Fushu No. 317 as determined by ultra performance liquid chromatography ( $\mu \mathrm{g} / \mathrm{g}$ fresh weight, $\mathrm{FW})$.

\begin{tabular}{|c|c|c|c|c|c|c|}
\hline \multirow{2}{*}{ Identity $^{a}$} & \multicolumn{3}{|c|}{ Fushu No. 23} & \multicolumn{3}{|c|}{ Fushu No. 317} \\
\hline & Lf1 & Lf2 & Lf3 & Lf1 & Lf2 & Lf3 \\
\hline Су 3-soph-5glc & $0.93 \pm 0.22$ & $1.65 \pm 0.10$ & $0.58 \pm 0.06$ & $3.47 \pm 0.36$ & $1.36 \pm 0.17$ & $0.21 \pm 0.02$ \\
\hline Peo 3-soph-5glc & $0.68 \pm 0.36$ & $2.31 \pm 0.12$ & $1.40 \pm 0.21$ & $5.88 \pm 0.84$ & $2.76 \pm 0.60$ & $0.53 \pm 0.05$ \\
\hline Су 3-p-hydroxybenzoylsoph-5glc & $2.46 \pm 0.50$ & $6.66 \pm 0.39$ & $5.71 \pm 0.43$ & $10.41 \pm 0.10$ & $4.06 \pm 1.11$ & $0.63 \pm 0.06$ \\
\hline Cy 3-caffeylsophsoph-5glc & $0.14 \pm 0.00$ & $0.40 \pm 0.07$ & $0.79 \pm 0.11$ & $11.09 \pm 1.14$ & $4.55 \pm 1.08$ & $0.31 \pm 0.02$ \\
\hline Peo 3-p-hydroxybenzoylsoph-5glc & $0.98 \pm 0.49$ & $3.55 \pm 0.11$ & $2.85 \pm 0.22$ & $5.72 \pm 0.05$ & $2.76 \pm 1.00$ & $0.45 \pm 0.08$ \\
\hline Peo 3-caffeylsophsoph-5glc & $0.02 \pm 0.00$ & $0.11 \pm 0.02$ & $0.18 \pm 0.01$ & $5.45 \pm 1.16$ & $2.00 \pm 0.67$ & $0.14 \pm 0.01$ \\
\hline Су 3-p-coumarylsoph-5glc & $0.06 \pm 0.01$ & $0.17 \pm 0.04$ & $0.12 \pm 0.01$ & $21.23 \pm 2.04$ & $7.42 \pm 1.91$ & $0.55 \pm 0.05$ \\
\hline Сy 3-feruloylsoph-5glc & $0.21 \pm 0.01$ & $0.38 \pm 0.03$ & $0.45 \pm 0.08$ & $4.65 \pm 0.72$ & $1.63 \pm 0.19$ & $0.31 \pm 0.05$ \\
\hline Peo 3-p-coumarylsoph-5glc & $0.11 \pm 0.01$ & $0.42 \pm 0.07$ & $0.31 \pm 0.01$ & $15.42 \pm 0.79$ & $6.84 \pm 2.22$ & $0.35 \pm 0.04$ \\
\hline Peo 3-feruloylsoph-5glc & $0.05 \pm 0.03$ & $0.18 \pm 0.04$ & $0.20 \pm 0.01$ & $4.50 \pm 0.85$ & $1.45 \pm 0.35$ & $0.33 \pm 0.06$ \\
\hline Су 3-dicaffeylsoph-5glc & $0.24 \pm 0.07$ & $0.71 \pm 0.13$ & $3.27 \pm 0.66$ & $11.08 \pm 0.82$ & $6.34 \pm 2.36$ & $0.11 \pm 0.01$ \\
\hline Су 3-caffeoyl-p-hydroxybenzoylsoph-5glc & $1.43 \pm 0.28$ & $4.04 \pm 0.61$ & $8.57 \pm 1.54$ & $4.52 \pm 0.40$ & $2.61 \pm 0.95$ & $0.09 \pm 0.01$ \\
\hline Cy 3-caffeoyl-p-coumarylsoph-5glc & $0.02 \pm 0.00$ & $0.10 \pm 0.03$ & $0.16 \pm 0.04$ & $7.24 \pm 0.53$ & $4.18 \pm 1.27$ & $0.05 \pm 0.00$ \\
\hline Су 3-caffeoyl-feruloylsoph-5glc & $0.06 \pm 0.03$ & $0.21 \pm 0.02$ & $0.70 \pm 0.22$ & $5.07 \pm 0.69$ & $2.14 \pm 0.40$ & $0.10 \pm 0.00$ \\
\hline Peo 3-dicaffeoylsoph-5glc & $0.02 \pm 0.00$ & $0.09 \pm 0.01$ & $0.40 \pm 0.03$ & $4.81 \pm 0.35$ & $2.72 \pm 1.11$ & $0.04 \pm 0.00$ \\
\hline Peo 3-caffeoyl-p-hydroxybenzoylsoph-5glc & $0.20 \pm 0.09$ & $0.88 \pm 0.08$ & $1.56 \pm 0.06$ & $2.31 \pm 0.14$ & $1.42 \pm 0.61$ & $0.04 \pm 0.00$ \\
\hline Peo 3-caffeoyl- $p$-coumarylsoph-5glc & - & $0.02 \pm 0.00$ & $0.02 \pm 0.00$ & $2.57 \pm 0.28$ & $2.12 \pm 0.67$ & $0.01 \pm 0.00$ \\
\hline Peo 3-caffeoyl-feruloylsoph-5glc & - & $0.04 \pm 0.01$ & $0.14 \pm 0.02$ & $1.92 \pm 0.45$ & $0.85 \pm 0.24$ & $0.03 \pm 0.00$ \\
\hline Total anthocyanin & $7.62 \pm 1.43$ & $21.92 \pm 1.25$ & $27.41 \pm 2.69$ & $127.33 \pm 7.55$ & $57.20 \pm 6.75$ & $4.28 \pm 0.25$ \\
\hline non-acylated anthocyanin & $0.98 \pm 0.24$ & $1.84 \pm 0.14$ & $0.78 \pm 0.06$ & $7.97 \pm 1.21$ & $2.81 \pm 0.51$ & $0.53 \pm 0.09$ \\
\hline monoacylated anthocyanin & $6.01 \pm 1.75$ & $17.98 \pm 1.48$ & $23.46 \pm 3.42$ & $60.99 \pm 4.58$ & $29.39 \pm 8.78$ & $2.28 \pm 0.24$ \\
\hline diacylated anthocyanin & $0.63 \pm 0.13$ & $2.10 \pm 0.25$ & $3.17 \pm 0.21$ & $58.37 \pm 5.93$ & $25.00 \pm 7.62$ & $1.48 \pm 0.17$ \\
\hline cyanidin-based anthocyanin & $5.59 \pm 1.59$ & $15.66 \pm 0.93$ & $12.38 \pm 1.11$ & $83.32 \pm 7.19$ & $33.37 \pm 8.95$ & $3.47 \pm 0.38$ \\
\hline peonidin-based anthocyanin & $2.03 \pm 0.52$ & $6.26 \pm 0.94$ & $15.03 \pm 2.58$ & $44.01 \pm 4.52$ & $23.83 \pm 7.96$ & $0.81 \pm 0.11$ \\
\hline
\end{tabular}

${ }^{\mathrm{a}} \mathrm{Cy}=$ cyanidin, $\mathrm{Peo}=$ peonidin, $\mathrm{soph}=$ sophorside, glc $=$ glucoside.

\subsection{Differential Accumulation of Anthocyanins in the Leaves of the Cultivars Fushu No. 23 and Fushu No. 317}

As shown in Figure 1, the anthocyanin accumulation in the leaves of the cultivars Fushu No. 23 and Fushu No. 317 was different. The anthocyanin content in Fushu No. 23 leaves increased with the growth of the first to third leaves (one- to seven-day-old from new leaves), while the anthocyanin content in Fushu No. 317 leaves decreased in that period.

The total anthocyanin content in sweet potato leaves ranged from 4.28 to $127.33 \mu \mathrm{g} / \mathrm{g}$ fresh weight (FW). The accumulation of anthocyanin monomers in the leaves of Fushu No. 23 and Fushu No. 317 was different (Table 2). In Fushu No. 23, the simple structure of anthocyanidin monomers led cyanidin 3-sophoroside-5-glucoside to increase from the first to second leaves, and then decrease from the second to third leaves. Meanwhile, the structurally complex monomers in Fushu No. 317 caused cyanidin 3-(6', $6^{\prime \prime}$-dicaffeylsophoroside)-5-glucoside to increase from the first to the third leaves.

\subsection{Expression of Anthocyanidin Biosynthesis Structural Genes and Transcription Factor}

The synthesis precursor of anthocyanin monomers in sweet potato leaves is phenylalanine, and structural genes in the synthesis process include: phenylalanine ammonialyase $(P A L)$, cinnamic acid-4-hydroxylase (C4L), 4-coumarate:CoA ligase (4CL), chalconesynthase $(C H S)$, chalconeisomerase $(C H I)$, flavanone 3-hydroxylase $(F 3 H)$, flavonoids $3^{\prime}$-hydroxylase $\left(F 3^{\prime} H\right)$, dihydrofavonol4-reductase $(D F R)$, and anthocyanin synthase (ANS) [21]. The first anthocyanin monomer was cyanidin, and other acylated anthocyanin monomers formed under the action of the UDP-glucose flavonoid 3-O-glucosyltransferase (UFGT) [22] or anthocyanin 3-O-acyltransferases (3AT) [23,24] genes (Figure 3). DFRs, UFGTs, and $3 A t s$ are mined from two diploid wild relatives of cultivated sweet potato (Ipomoea triloba. L). UFGT1 (3GGT) was found to transfer glucose to glycosylated anthocyanins in purple sweet potato [25], but this is the only UFGT whose function in the sweet potato has been established. The transcription factors in anthocyanin synthesis were $M Y B, b L H L$, and $W D R 40[21,26] . I b M Y B 1$ is a key regulatory gene of anthocyanin biosynthesis in the storage roots of purple-fleshed sweet potato [27]. IbMYB60 is homologous to the Arabidopsis R2R3-MYB transcription factor AtMYB60, a transcriptional 
repressor of anthocyanin biosynthesis in lettuce [28]. AtMYB75 (PAP1) [29,30] and AtMYB113 (PAP2) [31,32] regulate the anthocyanin pathway in Arabidopsis seedlings. Two homologous AtMYB75 genes (IbMYB75-1 and IbMYB75-2) were mined from Ipomoea triloba genome, but only one homologous gene IbMYB113 was found in the Ipomoea triloba genome (www.sweetpotato-garden.kazusa.or.jp).

\begin{tabular}{|c|c|c|c|c|c|c|c|}
\hline \multirow{3}{*}{$\begin{array}{r}\text { Phenylalanine } \\
\downarrow \text { PAL }\end{array}$} & \multirow{2}{*}{$\begin{array}{c}\text { Related } \\
\text { Genes }\end{array}$} & \multicolumn{6}{|c|}{ Fushu No.23/Fushu No.317 expression ratio } \\
\hline & & Lf1 & $p$-value & Lf2 & $p$-value & Lf3 & $p$-value \\
\hline & $P A L$ & 3.27 & 0.0060 & 5.08 & 0.0236 & 2.43 & 0.0060 \\
\hline$\downarrow \mathrm{C} 4 \mathrm{H}$ & $4 C L$ & 0.77 & 0.1506 & 2.80 & 0.0011 & 1.11 & 0.0528 \\
\hline p-Coumaric acid & $\mathrm{C} 4 \mathrm{H}$ & 16.07 & 0.0004 & 9.16 & 0.0206 & 4.87 & 0.0008 \\
\hline$\downarrow 4 \mathrm{CL}$ & CHS & 12.68 & 0.0014 & 2.64 & 0.0027 & 78.72 & 0.0004 \\
\hline 4-Coumaroyl-CoA & $\mathrm{CHI}$ & 2.42 & 0.0029 & 1.22 & 0.0308 & 6.92 & 0.0050 \\
\hline 5 & DFR1 & 5.10 & 0.0009 & 5.80 & 0.0040 & 2.68 & 0.0112 \\
\hline Chalcone & DFR2 & 3.44 & 0.0001 & 2.23 & 0.0193 & 1.46 & 0.0082 \\
\hline$\downarrow \mathrm{CHI}$ & DFR3 & 0.03 & 0.0001 & 2.34 & 0.0012 & 2.66 & 0.0013 \\
\hline Naringenin flavanone & DFR4 & 9.44 & 0.0004 & 1.25 & 0.0126 & 32.72 & 0.0009 \\
\hline$\downarrow$ F3H, F3'H & F3H1 & 1.40 & 0.0130 & 2.46 & 0.0001 & 9.70 & 0.0001 \\
\hline Dihydroflavonols & $\mathrm{F3H2}$ & 0.17 & 0.0010 & 2.73 & 0.0032 & 1.23 & 0.0430 \\
\hline$\downarrow \quad$ DFR & $F 3^{\prime} H$ & 3.19 & 0.0418 & 1.00 & 0.0208 & 25.88 & 0.0025 \\
\hline Leucoanthocyanins & $A N S$ & 0.08 & 0.2692 & 0.88 & 0.0560 & 11.21 & 0.0016 \\
\hline$\downarrow$ ANS & $U F G T 1$ & 0.08 & 0.0002 & 1.31 & 0.1776 & 0.47 & 0.0001 \\
\hline & UFGT2 & 0.01 & 0.0001 & 2.75 & 0.0005 & 0.65 & 0.0720 \\
\hline Antnocyanioms & UFGT3 & 1.85 & 0.0003 & 0.87 & 0.0163 & 16.21 & 0.0004 \\
\hline Cyaniom et al. & UFGT4 & 0.05 & 0.0003 & 1.16 & 0.2244 & 0.35 & 0.0001 \\
\hline$\downarrow \quad$ UFGT & $3 A T 1-1$ & 0.04 & 0.0005 & 2.37 & 0.0008 & 1.73 & 0.0001 \\
\hline Anthocyanidins & $3 A T 1-2$ & 0.01 & 0.0001 & 1.92 & 0.0219 & 2.95 & 0.0038 \\
\hline Cyanidin 3-O-Glycoside et al. & $3 A T 1-3$ & 0.12 & 0.0001 & 3.22 & 0.0002 & 1.68 & 0.0035 \\
\hline $3 \mathbf{A}$ & $3 A T 1-4$ & 0.01 & 0.0008 & 0.92 & 0.0485 & 0.45 & 0.0003 \\
\hline$\checkmark$ JAI & $3 A T 1-5$ & 0.01 & 0.0008 & 1.16 & 0.0485 & 0.45 & 0.0003 \\
\hline Anthocyanidins & $3 A T 1-6$ & 1.85 & 0.0031 & 1.48 & 0.0072 & 2.10 & 0.0050 \\
\hline Cyanidin 3-O-p-coumarylglycoside et & $3 A T 1-7$ & 0.02 & 0.0007 & 5.05 & 0.0002 & 1.51 & 0.0333 \\
\hline al. & $3 A T 2$ & 0.03 & 0.0002 & 2.28 & 0.0089 & 0.83 & 0.1045 \\
\hline
\end{tabular}

(a)

Figure 3. Cont. 


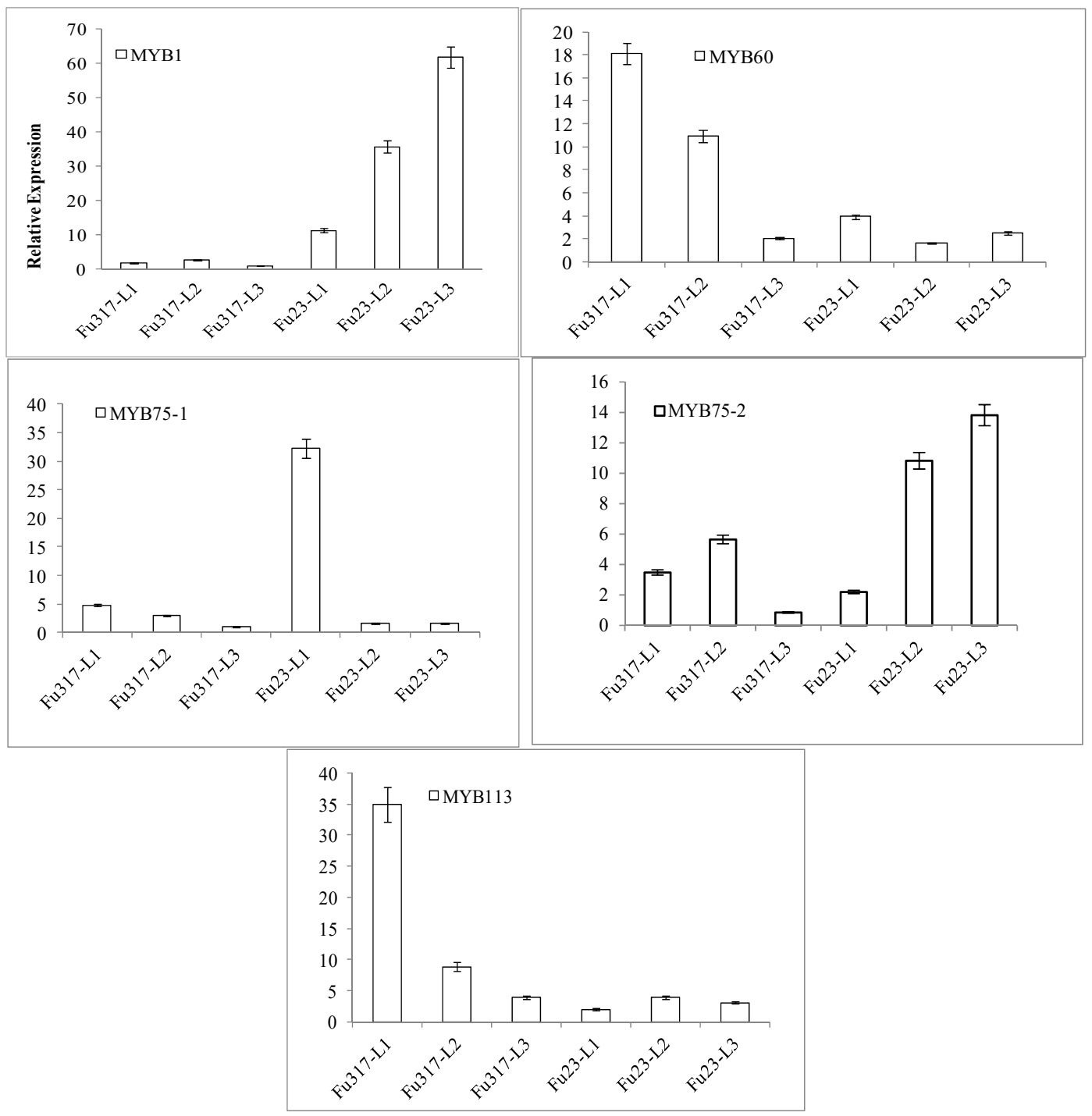

(b)

Figure 3. Expression of anthocyanin structural genes and transcription factors in the purple sweet potato cultivars Fushu No. 23 and Fushu No. 317. (a) Simplified model of the anthocyanin biosynthetic pathway and qRT-PCR analysis of the transcript abundance of the anthocyanin biosynthesis-related genes in Fushu No. 23 and Fushu No. 317 leaves. (b) Expression of transcription factors IbMYBs in sweet potato leaves.

To further clarify the internal connection of anthocyanin content with transcription levels of the genes involved in anthocyanin biosynthesis, 25 anthocyanin structural genes and five transcription factor genes were detected by quantitative real-time PCR. We found there were substantial differences in the expression of anthocyanidin-related genes in Fushu No. 23 and Fushu No. 317, and we found that the two cultivars had an opposite accumulation pattern. From the first to third leaves of Fushu No. 317, CHS, DFRs, F3Hs, ANS, UFGTs, and 3Ats had a tendency to first increase and then decrease. Most anthocyanin biosynthesis related-myb transcription factors also had the same pattern. However, DFRs were correlated with the increase in anthocyanin content in the first to third leaves of Fushu No. 23.

On comparing the first leaf of Fushu No. 23 to Fushu No. 317, anthocyanin content was greater in Fushu No. 317 than Fushu No. 23, while the expression of ANS in the leaf of Fushu No. 23 was lower than in Fushu No. 317. Therefore, we speculate that ANS is the key gene for anthocyanin biosynthesis in the first leaf of sweet potato. 
The anthocyanin content in the third leaf was also different. Expression of the structural genes: CHS, DFR4, F3'H1, ANS, and UFGT3 were greater in Fushu No. 23 than in Fushu No. 317. Therefore, CHS, DFR4, F3'H1, ANS, and UFGT3 may be vital regulatory genes of anthocyanin biosynthesis in sweet potato leaves.

MYB transcription factors (TFs) have had a long association with anthocyanin biosynthesis. In sweet potato, activation of anthocyanin levels by IbMYB1 was first observed in a functional complementation experiment using a mutant. Mano et al. (2007) reported IbMYB1 was only expressed in the roots of purple-fleshed sweet potatoes but not in other related-tissues such as stems, leaves, flowers, and the roots of orange-, yellow-, or white-fleshed varieties [24], but we found IbMYB1 can be highly expressed in the leaves of sweet potato variety Fushu No. 23, and can have very low expression in the leaves of Fushu No. 317. Perhaps the biosynthesis of anthocyanins in the leaves of sweet potato is through more than one metabolic pathway. MYB60 and MYB113 decreased from the first to third leaves, while $M Y B 1$ and $M Y B 75-2$ increased from the first to third leaves, possibly due to the fact that MYB has different effects on anthocyanin biosynthesis in different sweet potato leaves.

\subsection{Anthocyanins in Roots of Purple-fleshed Sweet Potato Were Different from the Anthocyanins in Sweet Potato Leaves}

In order to further determine differences in the composition of anthocyanins between sweet potato leaves and roots, we used the same method to analyze the anthocyanin components of five sweet potato leaves and five sweet potato roots. Fushu No. 317, Fushu No. 25, and OP contained 18 anthocyanin components and Fushu No. 23 and Yanshu No. 5 contained 17 anthocyanin components. However, cyanidin 3-p-coumarylsophoroside-5-glucoside and cyanidin 3-caffeoyl-p-coumaryl sophoroside-5-glucoside were not found in the any of the roots in this study. Interestingly, Tian et al. (2005) found cyanidin 3-p-coumarylsophoroside-5-glucoside and cyanidin 3-caffeoyl-p-coumaryl sophoroside-5-glucoside in a PL (purple line) cell line generated from the storage root of the purple-fleshed sweet potato cultivar Ayamurasaki [4], but this has not been identified in any earlier reports on purple-fleshed sweet potato [9,33]. It is possible that these compounds were not found in our study because they existed at such a low level that they could not be detected.

In this study, among five purple-fleshed sweet potatoes in this study, Longzishu No. 6 had the greatest number of anthocyanin monomers in the roots. The total contents of anthocyanins in Longzishu No. 6 roots were $255.75 \pm 17.39 \mu \mathrm{g} / \mathrm{g}$ FW (Table 3), not very high. Anthocyanin monomers cyanidin 3-p-coumarylsophoroside-5-glucoside, cyanidin 3-caffeoyl-p-coumaryl sophoroside-5-glucoside, and cyanidin 3-caffeoyl-feruloylsophoroside-5-glucoside were not found in the roots of Fushu No. 317. The total contents of anthocyanins in Fushu No. 317 leaves $(188.81 \pm 18.35 \mu \mathrm{g} / \mathrm{g}$ FW) were much lower than that in the roots $(1009.29 \pm 66.41 \mu \mathrm{g} / \mathrm{g} \mathrm{FW})$. As Su et al. speculated [11], maybe anthocyanin biosynthesis between sweet potato leaves and roots involved different phenotypes. Fushu No. 9 lacked two anthocyanin monomers: peonidin 3-p-coumarylsophroside-5-glucoside and peonidin 3-caffeoyl-p-coumarylsophoroside-5-glucoside in roots. Fushu No. 24 and Longzishu No. 4 only contained 13 anthocyanin monomers in the roots. It should be noted that the total anthocyanins in the roots of purple-fleshed sweet potato had greater changes with different varieties, but the total anthocyanins in the leaves of sweet potato showed small changes. A future study to explain the genotype of anthocyanin biosynthesis genes in the leaves and roots of sweet potato may be necessary. 
Table 3. Quantity of anthocyanins (1-14) from the leaves and roots of different sweet potato cultivars ( $\mu \mathrm{g} / \mathrm{g}$ FW).

\begin{tabular}{|c|c|c|c|c|c|c|c|c|c|c|}
\hline \multirow{2}{*}{ Identity ${ }^{a}$} & \multicolumn{5}{|c|}{ Leaves } & \multicolumn{5}{|c|}{ Roots } \\
\hline & Fushu No. 23 & Fushu No. 317 & Fushu No. 25 & OP & Yanshu No. 5 & Fushu No. 9 & Fushu No. 24 & Longzishu No. 4 & Longzishu No. 6 & Fushu No. 317 \\
\hline Су 3-soph-5glc & $3.16 \pm 0.27$ & $5.04 \pm 0.62$ & $4.27 \pm 0.32$ & $7.51 \pm 0.46$ & $3.75 \pm 0.26$ & $2.82 \pm 0.16$ & $3.60 \pm 0.25$ & $1.30 \pm 0.09$ & $3.21 \pm 0.32$ & $42.02 \pm 2.54$ \\
\hline Peo 3-soph-5glc & $4.38 \pm 0.46$ & $9.16 \pm 0.94$ & $2.62 \pm 0.15$ & $5.38 \pm 0.32$ & $8.15 \pm 0.52$ & $4.82 \pm 0.25$ & $4.02 \pm 0.35$ & $5.13 \pm 0.69$ & $27.41 \pm 1.86$ & $57.40 \pm 3.68$ \\
\hline Су 3-p-hydroxybenzoylsoph-5glc & $14.83 \pm 1.98$ & $15.09 \pm 2.21$ & $3.58 \pm 0.16$ & $2.55 \pm 0.15$ & $1.27 \pm 0.08$ & $2.87 \pm 0.12$ & $3.41 \pm 0.27$ & $3.24 \pm 0.73$ & $12.62 \pm 0.79$ & $112.39 \pm 8.52$ \\
\hline Су 3-caffeylsophsoph-5glc & $1.33 \pm 0.15$ & $15.94 \pm 2.66$ & $14.31 \pm 0.86$ & $16.64 \pm 1.12$ & $14.19 \pm 1.03$ & $1.72 \pm 0.08$ & $7.51 \pm 0.43$ & $16.15 \pm 0.89$ & $27.93 \pm 1.56$ & $19.97 \pm 1.64$ \\
\hline Peo 3-p-hydroxybenzoylsoph-5glc & $7.39 \pm 0.66$ & $8.93 \pm 1.04$ & $5.10 \pm 0.35$ & $12.33 \pm 0.84$ & $6.17 \pm 0.46$ & $1.12 \pm 0.07$ & $13.84 \pm 0.95$ & $1.65 \pm 0.06$ & $4.91 \pm 0.23$ & $135.07 \pm 9.36$ \\
\hline Peo 3-caffeylsophsoph-5glc & $0.31 \pm 0.06$ & $7.58 \pm 0.98$ & $3.70 \pm 0.21$ & $10.47 \pm 0.76$ & $6.75 \pm 0.81$ & $0.99 \pm 0.11$ & $0.77 \pm 0.06$ & - & $3.67 \pm 0.28$ & $13.28 \pm 1.12$ \\
\hline Су 3-p-coumarylsoph-5glc & $0.34 \pm 0.07$ & $29.20 \pm 1.45$ & $22.26 \pm 2.00$ & $40.33 \pm 2.38$ & $20.16 \pm 1.74$ & - & - & - & - & - \\
\hline Cy 3-feruloylsoph-5glc & $1.04 \pm 0.15$ & $6.59 \pm 0.73$ & $8.08 \pm 0.19$ & $9.10 \pm 0.55$ & $5.87 \pm 0.44$ & $11.46 \pm 1.34$ & $1.21 \pm 0.08$ & $8.53 \pm 0.77$ & $19.29 \pm 1.64$ & $11.32 \pm 0.88$ \\
\hline Peo 3-p-coumarylsoph-5glc & $0.84 \pm 0.11$ & $22.61 \pm 1.56$ & $27.73 \pm 1.87$ & $31.23 \pm 2.53$ & $15.62 \pm 1.15$ & - & $4.15 \pm 0.41$ & $2.81 \pm 0.16$ & $8.47 \pm 0.53$ & $51.15 \pm 3.63$ \\
\hline Peo 3-feruloylsoph-5glc & $0.43 \pm 0.05$ & $6.27 \pm 0.42$ & $7.69 \pm 0.23$ & $8.67 \pm 0.79$ & $5.58 \pm 0.65$ & $19.71 \pm 1.74$ & $3.47 \pm 0.26$ & $7.84 \pm 0.63$ & $61.44 \pm 3.86$ & $17.28 \pm 1.41$ \\
\hline Cy 3-dicaffeylsoph-5glc & $4.23 \pm 0.51$ & $17.53 \pm 2.08$ & $21.49 \pm 1.68$ & $24.21 \pm 1.82$ & $12.1 \pm 1.04$ & $1.04 \pm 0.07$ & $3.11 \pm 0.22$ & $16.72 \pm 0.92$ & $14.07 \pm 1.12$ & $41.56 \pm 3.76$ \\
\hline Су 3-caffeoyl-p-hydroxybenzoylsoph-5glc & $14.04 \pm 2.11$ & $7.22 \pm 0.55$ & $8.85 \pm 0.66$ & $9.97 \pm 0.73$ & $6.42 \pm 0.47$ & $3.27 \pm 0.19$ & - & $3.04 \pm 0.23$ & $3.99 \pm 0.24$ & $32.23 \pm 2.09$ \\
\hline Cy 3-caffeoyl- $p$-coumarylsoph-5glc & $0.28 \pm 0.05$ & $11.48 \pm 0.92$ & $14.07 \pm 1.12$ & $15.85 \pm 1.75$ & $7.92 \pm 0.78$ & & & & & \\
\hline Cy 3-caffeoyl-feruloylsoph-5glc & $0.97 \pm 0.10$ & $7.30 \pm 0.67$ & $5.06 \pm 0.36$ & $10.09 \pm 0.95$ & $6.50 \pm 0.47$ & $9.15 \pm 1.22$ & $0.91 \pm 0.04$ & $17.33 \pm 0.81$ & $3.77 \pm 0.13$ & $82.11 \pm 5.49$ \\
\hline Peo 3-dicaffeoylsoph-5glc & $0.50 \pm 0.07$ & $7.58 \pm 0.72$ & $6.37 \pm 0.43$ & $10.47 \pm 1.07$ & $5.23 \pm 0.32$ & $13.07 \pm 1.68$ & $10.63 \pm 0.78$ & $40.75 \pm 2.58$ & $63.36 \pm 4.75$ & $37.02 \pm 1.83$ \\
\hline Peo 3-caffeoyl-p-hydroxybenzoylsoph-5glc & $2.64 \pm 0.33$ & $3.77 \pm 0.21$ & $8.26 \pm 0.61$ & $5.21 \pm 0.33$ & $3.36 \pm 0.23$ & $1.52 \pm 0.13$ & $1.00 \pm 0.03$ & $18.17 \pm 0.94$ & $1.62 \pm 0.08$ & $166.51 \pm 10.25$ \\
\hline Peo 3-caffeoyl- $p$-coumarylsoph-5glc & $0.04 \pm 0.01$ & $4.70 \pm 0.37$ & $5.76 \pm 0.16$ & $6.49 \pm 0.49$ & $3.25 \pm 0.28$ & & & - & - & $181.75 \pm 9.58$ \\
\hline Peo 3-caffeoyl-feruloylsoph-5glc & $0.18 \pm 0.02$ & $2.80 \pm 0.22$ & $3.43 \pm 0.04$ & $3.87 \pm 0.22$ & $2.49 \pm 0.17$ & $3.75 \pm 0.26$ & - & - & - & $8.22 \pm 0.63$ \\
\hline Total anthocyanin & $56.95 \pm 7.10$ & $188.81 \pm 18.35$ & $172.65 \pm 11.41$ & $230.36 \pm 17.26$ & $134.8 \pm 9.65$ & $77.32 \pm 7.42$ & $57.64 \pm 4.13$ & $142.66 \pm 9.50$ & $255.75 \pm 17.39$ & $1009.29 \pm 66.41$ \\
\hline
\end{tabular}

${ }^{a} \mathrm{Cy}=$ cyanidin, $\mathrm{Peo}=$ peonidin, $\mathrm{soph}=$ sophorside, glc $=$ glucoside 
Supplementary Materials: The following are available online, Figure S1: UPLC-QTOF-MS/MS of anthocyanins in two purple sweetpotato leaves, Table S1: Primer sequences used for detection of genes related to anthocyanin biosynthesis by qRT-PCR.

Author Contributions: Conceptualization, G.L. and S.Q.; methodology, G.L., Z.L. (Zhaomiao Lin) and H.L.; software, G.L.; validation, G.L. and H.Z.; formal analysis, G.L.; investigation, Y.X.; resources, Z.L. (Zhonghua Liu), R.J., W.L. and Y.Q.; data curation, G.L; writing—original draft preparation, G.L.; writing-review and editing, G.L., Z.L. (Zhaomiao Lin), H.Z. and G.X.; visualization, Z.L. (Zhonghua Liu), R.J., W.L. and Y.Q.; supervision, Y.Q. and H.T.; project administration, G.L. and S.Q.; funding acquisition, S.Q. and H.T.

Funding: This work was supported by Natural Science Foundation of Fujian Province of China (2018J01046), Special Fund for the Industrial Technology System Construction of Modern Agriculture of China (CARS-10-B14) and Fujian Academy of Agricultural Sciences Research Project (DC2017-7).

Acknowledgments: We thank LetPub (www.letpub.com) for its linguistic assistance during the preparation of this manuscript.

Conflicts of Interest: The authors declare no conflict of interest.

\section{Abbreviations}

$\begin{array}{ll}\text { PAL } & \text { phenylalanine ammonialyase } \\ \text { C4L } & \text { cinnamic acid-4-hydroxylase } \\ \text { 4CL } & \text { 4-coumarate:CoA ligase } \\ \text { CHS } & \text { chalconesynthase } \\ \text { CHI } & \text { chalconeisomerase } \\ \text { F3H } & \text { flavanone 3-hydroxylase } \\ \text { F3'H } & \text { flavonoids 3'-hydroxylase } \\ \text { DFR } & \text { dihydrofavonol 4-reductase } \\ \text { ANS } & \text { anthocyanin synthase } \\ \text { UFGT } & \text { UDP-glucose flavonoid 3-O-glucosyltransferase } \\ \text { 3AT } & \text { anthocyanin 3-O-acyltransferases } \\ \text { qRT-PCR } & \text { quantitative real time polymerase chain reaction } \\ \text { SD } & \text { standard deviation } \\ \text { UPLC-QTOF-MS } & \text { ultra-performance liquid chromatography/quadrupole time-of-flight mass spectrometry } \\ \text { UPLC-DAD } & \text { ultra-performance liquid chromatography diode-array detection } \\ \text { FW } & \text { fresh weight }\end{array}$

\section{References}

1. Tahara, M. Current developments in breeding, genetics, genomics, and molecular biology applied to sweetpotato improvement. Breed. Sci. 2017, 67, 1. [CrossRef] [PubMed]

2. Tanaka, M.; Ishiguro, K.; Oki, T.; Okuno, S. Functional components in sweetpotato and their genetic improvement. Breed. Sci. 2017, 67, 52-61. [CrossRef] [PubMed]

3. Cai, N.T.; Huang, H.K.; Qiu, Y.X.; Zheng, X.; Wu, Q.Y.; Luo, W.B.; Li, G.X. Breeding and cultivate techniques fo new sweetpotato variety Fushu 7-6 used as leaf vegetable. Fujian J. Agric. Sci. 2006, 1, 12-15.

4. Tian, Q.; Konczak, I.; Schwartz, S.J. Probing anthocyanin profiles in purple sweetpotato cell line (Ipomoea batatas L. Cv. Ayamurasaki) by high-performance liquid chromatography and electrospray ionization tandem mass spectrometry. J. Agric. Food Chem. 2005, 53, 6503-6509. [CrossRef] [PubMed]

5. Truong, V.-D.; Deighton, N.; Thompson, R.T.; McFeeters, R.F.; Dean, L.O.; Pecota, K.V.; Yencho, G.C. Characterization of Anthocyanins and Anthocyanidins in Purple-Fleshed Sweetpotatoes by HPLC-DAD/ESI-MS/MS. J. Agric. Food Chem. 2010, 58, 404-410. [CrossRef]

6. Lee, M.J.; Park, J.S.; Choi, D.S.; Jung, M.Y. Characterization and quantitation of anthocyanins in purple-fleshed sweetpotatoes cultivated in Korea by HPLC-DAD and HPLC-ESI-QTOF-MS/MS. J. Agric. Food Chem. 2013, 61, 3148-3158. [CrossRef]

7. Xu, J.; Su, X.; Lim, S.; Griffin, J.; Carey, E.; Katz, B.; Tomich, J.; Smith, J.S.; Wang, W. Characterisation and stability of anthocyanins in purple-fleshed sweetpotato P40. Food Chem. 2015, 186, 90-96. [CrossRef] 
8. He, W.; Zeng, M.; Chen, J.; Jiao, Y.; Niu, F.; Tao, G.; Zhang, S.; Qin, F.; He, Z. Identification and quantitation of anthocyanins in purple-fleshed sweetpotatoes cultivated in china by UPLC-PDA and UPLC-QTOF-MS/MS. J. Agric. Food Chem. 2016, 64, 171-177. [CrossRef]

9. Terahara, N.; Shimizu, T.; Kato, Y.; Nakamura, M.; Maitani, T.; Yamaguchi, M.-A.; Goda, Y. Six diacylated anthocyanins from the storage roots of purple sweetpotato, (Ipomoea batatas). Biosci. Biotechnol. Biochem. 1999, 63, 1420-1424. [CrossRef]

10. Islam, M.S.; Yoshimoto, M.; Terahara, N.; Yamakawa, O. Anthocyanin compositions in sweetpotato (Ipomoea batatas L.) leaves. Biosci. Biotechnol. Biochem. 2002, 66, 2483-2486. [CrossRef]

11. Su, X.Y.; Griffin, J.; Xu, J.W.; Ouyan, P.; Zhao, Z.H.; Wang, W.Q. Identification and quantification of anthocyanins in purple-fleshed sweet potato leaves. Heliyon 2019, 5, e01964. [CrossRef] [PubMed]

12. Vishnu, V.R.; Renjith, R.S.; Mukherjee, A.; Anil, S.R.; Sreekumar, J.; Jyothi, A.N. Comparative study on the chemical structure and in vitro antiproliferative activity of anthocyanins in purple root tubers and leaves of sweetpotato (Ipomoea batatas). J. Agric. Food Chem. 2019, 67, 2467-2475. [CrossRef] [PubMed]

13. Sun, H.; Zhang, P.; Zhu, Y.; Lou, Q.; He, S. Antioxidant and prebiotic activity of five peonidin-based anthocyanins extracted from purple sweetpotato (Ipomoea batatas (L.) Lam.). Sci. Rep. 2018, 8, 5018. [CrossRef] [PubMed]

14. Wang, W.; Li, J.; Wang, Z.; Gao, H.; Su, L.; Xie, J.; Chen, X.; Liang, H.; Wang, C.; Han, Y. Oral hepatoprotective ability evaluation of purple sweetpotato anthocyanins on acute and chronic chemical liver injuries. Cell Biochem. Biophys. 2014, 69, 539-548. [CrossRef]

15. Hwang, Y.P.; Choi, J.H.; Yun, H.J.; Han, E.H.; Kim, H.G.; Kim, J.Y.; Park, B.H.; Khanal, T.; Choi, J.M.; Chung, Y.C.; et al. Anthocyanins from purple sweetpotato attenuate dimethylnitrosamine-induced liver injury in rats by inducing Nrf2-mediated antioxidant enzymes and reducing COX-2 and iNOS expression. Food Chem. Toxicol. 2011, 49, 93-99. [CrossRef]

16. Zhao, J.G.; Yan, Q.Q.; Lu, L.Z.; Zhang, Y.Q. In vivo antioxidant, hypoglycemic, and anti-tumor activities of anthocyanin extracts from purple sweetpotato. Nutr. Res. Pract. 2013, 7, 359-365. [CrossRef]

17. Zhu, Y.; Bo, Y.; Wang, X.; Lu, W.; Wang, X.; Han, Z.; Qiu, C. The effect of anthocyanins on blood pressure: A prisma-compliant meta-analysis of randomized clinical trials. Medince (Baltim.) 2016, 95, e3380. [CrossRef]

18. Zhuang, J.; Lu, J.; Wang, X.; Wang, X.; Hu, W.; Hong, F.; Zhao, X.X.; Zheng, Y.L. Purple sweet potato color protects against high-fat diet-induced cognitive deficits through AMPK-mediated autophagy in mouse hippocampus. J. Nutr. Biochem. 2019, 65, 35-45. [CrossRef]

19. Zhang, Z.C.; Su, G.H.; Luo, C.L.; Pang, Y.L.; Wang, L.; Li, X.; Wen, J.H.; Zhang, J.L. Effects of anthocyanins from purple sweet potato (Ipomoea batatas L. cultivar Eshu No. 8) on the serum uric acid level and xanthine oxidase activity in hyperuricemic mice. Food Funct. 2015, 6, 3045-3055. [CrossRef]

20. Schmittgen, T.D.; Livak, K.J. Analyzing real-time PCR data by the comparative CT method. Nat. Protoc. 2008, 6, 1101-1108. [CrossRef]

21. Dixon, R.A.; Liu, C.; Jun, J.H. Metabolic engineering of anthocyanins and condensed tannins in plants. Curr. Opin. Biotechnol. 2013, 24, 329-335. [CrossRef] [PubMed]

22. Hu, C.; Gong, Y.; Jin, S.; Zhu, Q. Molecular analysis of a UDP-glucose: Flavonoid 3-O-glucosyltransferase (UFGT) gene from purple potato (Solanum tuberosum). Mol. Biol. Rep. 2011, 38, 561-567. [CrossRef]

23. Luo, J.; Nishiyama, Y.; Fuell, C.; Taguchi, G.; Elliott, K.; Hill, L.; Tanaka, Y.; Kitayama, M.; Yamazaki, M.; Bailey, P.; et al. Convergent evolution in the BAHD family of acyl transferases: Identification and characterization of anthocyanin acyl transferases from Arabidopsis thaliana. Plant J. Cell Mol. Biol. 2007, 50, 678-695. [CrossRef] [PubMed]

24. Unno, H.; Ichimaida, F.; Suzuki, H.; Takahashi, S.; Tanaka, Y.; Saito, A.; Nishino, T.; Kusunoki, M.; Nakayama, T. Structural and mutational studies of anthocyanin malonyltransferases establish the features of BAHD enzyme catalysis. J. Biol. Chem. 2007, 282, 15812-15822. [CrossRef] [PubMed]

25. Wang, H.; Fan, W.; Wu, Y.; Zhang, P.; Wang, C.; Yang, J.; Appelhagen, I. A novel glycosyltransferase catalyses the transfer of glucose to glucosylated anthocyanins in purple sweetpotato. J. Exp. Bot. 2018, 69, 5444-5459. [PubMed]

26. Xie, F.; Burklew, C.E.; Yang, Y.; Liu, M.; Xiao, P.; Zhang, B.; Qiu, D. De novo sequencing and a comprehensive analysis of purple sweetpotato (Ipomoea batatas L.) transcriptome. Planta 2012, 236, 101-113. [CrossRef] [PubMed] 
27. Mano, H.; Ogasawara, F.; Sato, K.; Higo, H.; Minobe, Y. Isolation of a regulatory gene of anthocyanin biosynthesis in tuberous roots of purple-fleshed sweetpotato. Plant Physiol. 2007, 143, 1252-1268. [CrossRef]

28. Park, J.S.; Kim, J.B.; Cho, K.J.; Cheon, C.I.; Sung, M.K.; Choung, M.G.; Roh, K.H. Arabidopsis R2R3-MYB transcription factor AtMYB60 functions as a transcriptional repressor of anthocyanin biosynthesis in lettuce (Lactuca sativa). Plant Cell Rep. 2008, 27, 985-994. [CrossRef]

29. Teng, S.; Keurentjes, J.; Bentsink, L.; Koornneef, M.; Smeekens, S. Sucrose-specific induction of anthocyanin biosynthesis in Arabidopsis requires the MYB75/PAP1 gene. Plant Physiol. 2005, 139, 1840-1852. [CrossRef]

30. Li, X.; Gao, M.J.; Pan, H.Y.; Cui, D.J.; Gruber, M.Y. Purple canola: Arabidopsis PAP1 increases antioxidants and phenolics in Brassica napus leaves. J. Agric. Food Chem. 2010, 58, 1639-1645. [CrossRef]

31. Gonzalez, A.; Zhao, M.; Leavitt, J.M.; Lloyd, A.M. Regulation of the anthocyanin biosynthetic pathway by the TTG1/bHLH/Myb transcriptional complex in Arabidopsis seedlings. Plant J. Cell Mol. Biol. 2008, 53, 814-827. [CrossRef] [PubMed]

32. Baudry, A.; Caboche, M.; Lepiniec, L. TT8 controls its own expression in a feedback regulation involving TTG1 and homologous MYB and bHLH factors, allowing a strong and cell-specific accumulation of flavonoids in Arabidopsis thaliana. Plant J. Cell Mol. Biol. 2006, 46, 768-779. [CrossRef] [PubMed]

33. Goda, Y.; Shimizu, T.; Kato, Y.; Nakamura, M.; Maitani, T.; Yamada, T.; Terahara, N.; Yamaguchi, M. Two acylated anthocyanins from purple sweet-potato. Phytochemistry 1997, 44, 183-186. [CrossRef]

Sample Availability: Samples of the compounds are not available from the authors. 